\title{
CEREBRAL SALT WASTING SYNDROME IN A PATIENT WITH PRIMARY CNS LYMPHOMA
}

\author{
Vit Prochazka**, Zuzana Kubova ${ }^{\mathrm{a}}$, Ludek Raida ${ }^{\mathrm{a}}$, Tomas Papajik ${ }^{\mathrm{a}}$, Boris Paucek ${ }^{\mathrm{b}}$, \\ Karel Indrak ${ }^{\mathrm{a}}$
}

\author{
a Department of Hemato-Oncology, University Hospital, I.P. Pavlova 6, 77520 Olomouc, Czech Republic \\ b Medihope Magnetic Imaging Center, Prostejov, Czech Republic \\ e-mail:prochazv@fnol.cz
}

Received: February 23, 2009; Accepted: July 27, 2009

Key words: Cerebral salt wasting/Lymphoma/Hyponatremia

Background: In patients with severe central nervous system (CNS) diseases, life-threatening hyponatremia results from two main causes: the syndromes of inappropriate secretion of antidiuretic hormone (SIADH) and cerebral salt wasting (CSW). Clinical manifestations of the two conditions may be similar but their pathogeneses are fundamentally diverse. Distinguishing SIADH from CSW is based on the differential diagnoses of dilutional hyponatremia due to an excessive amount of water in the body (SIADH) and depletional hyponatremia caused by extensive natriuresis (CSW).

Methods and Results: We report the case of a 48-year-old, previously healthy male with a diffuse large B-cell lymphoma affecting the area of the basal ganglia and the right part of the thalamus and oppressing the third brain ventricle. After admission to the Department of Hemato-oncology, the patient was soporous and laboratory tests revealed severe serum hyponatremia, hypochloremia, hypoosmolality and marked polyuria with normal serum levels of urea, creatinine, glucose and calcium. Urinalysis showed high specific gravity of the urine and extreme wasting of sodium, chloride and kalium ions. Overall fluid balance was negative. After the first chemotherapy cycle, the CT scan confirmed regression of the tumour site. This was accompanied by patient's improved cognition as well as decreased polyuria and urine electrolyte excretion. Eventually, it was concluded that the diagnosis was cerebral salt wasting syndrome.

Conclusion: We noted a so far unpublished case of a 48-year-old male with the diagnosis of primary CNS lymphoma (DLBCL) affecting deep structures of the brain and associated with CSW.

Inadequate, otherwise inexplicable loss of sodium and chloride ions into the urine was first described in 1950 by Peters and colleagues in patients with organic CNS involvement ${ }^{1}$. Currently, the condition is referred to as a cerebral salt wasting syndrome (CSW) described in patients with brain tumours, traumas, intracranial haemorrhage, ischemic strokes and CNS infections ${ }^{2,3,4}$. Although its pathophysiology is not completely understood, increased natriuretic peptides from damaged nerve tissues and decreased sympathoadrenal kidney innervation are assumed ${ }^{5}$. Both mechanisms lead to severe hyponatremia, hypoosmolality and extracellular fluid volume depletion. The diagnosis is based on excluding other causes of increased natriuresis, such as e.g. mineralocorticoid deficiency or administration of diuretics ${ }^{6}$. The differential diagnostics of CSW versus syndrome of inappropriate secretion of antidiuretic hormone (SIADH) rests upon distinguishing between hyponatremic hyperhydration - and thus an excess of clear water - (SIADH) and hyponatremic dehydration $(\mathrm{CSW})^{7,8}$. Unlike SIADH management requiring strict fluid restriction and diuretic therapy, the treatment of CSW aims at achieving an adequate fluid balance and substitution of hyponatremia ${ }^{9}$. In some observational studies CSW has been found more frequently than SIADH. Most of the reports we have found in the literature come from neuroanaesthesia and neurocritical care units, but there is little evidence about its prevalence in non-critical neurology patients 9 .

We report the case of a 48-year-old, previously healthy male with a recent history of a behaviour disorder who was admitted to a psychiatric department. A CT scan of the brain verified a tumour sized $20 * 23 * 10 \mathrm{~mm}$ affecting the area of the basal ganglia and the right part of the thalamus and oppressing the third brain ventricle. CT-guided stereotactic biopsy of the tumor (Fig. 1) of the site and contrast magnetic resonance imaging (Fig. 2) were indicated. Histologic examination of the brain tissue biopsy revealed a diffuse infiltration with middle to large sized lymphocytes (immunophenotype CD 20+, bcl-2+, Ki-67+ in $>80 \%$ cells, CD 10-). The diagnosis of primary diffuse large B-cell lymphoma of the brain was established. Further staging evaluation showed no generalized disease. After admission to the Department of Hemato-oncology, the patient was soporous (Glasgow coma scale 7) and laboratory tests revealed severe serum hyponatremia (113 mmol/1, normal range 130-144 mmol/1), hypochloremia ( $81 \mathrm{mmol} / 1$, normal 95-110 $\mathrm{mmol} / 1$ ), serum hypoosmolality (244 mmol/kg, normal $275-300 \mathrm{mmol} / \mathrm{kg}$ ) and marked polyuria $(9600 \mathrm{ml} /$ day $)$, with normal serum levels of urea, creatinine, glucose and calcium. Urinalysis showed high specific gravity of the urine $\left(1035 \mathrm{~kg} / \mathrm{m}^{3}\right)$ and extreme wasting of $\mathrm{Na}^{+}(1116 \mathrm{mmol} / \mathrm{day}$, normal 


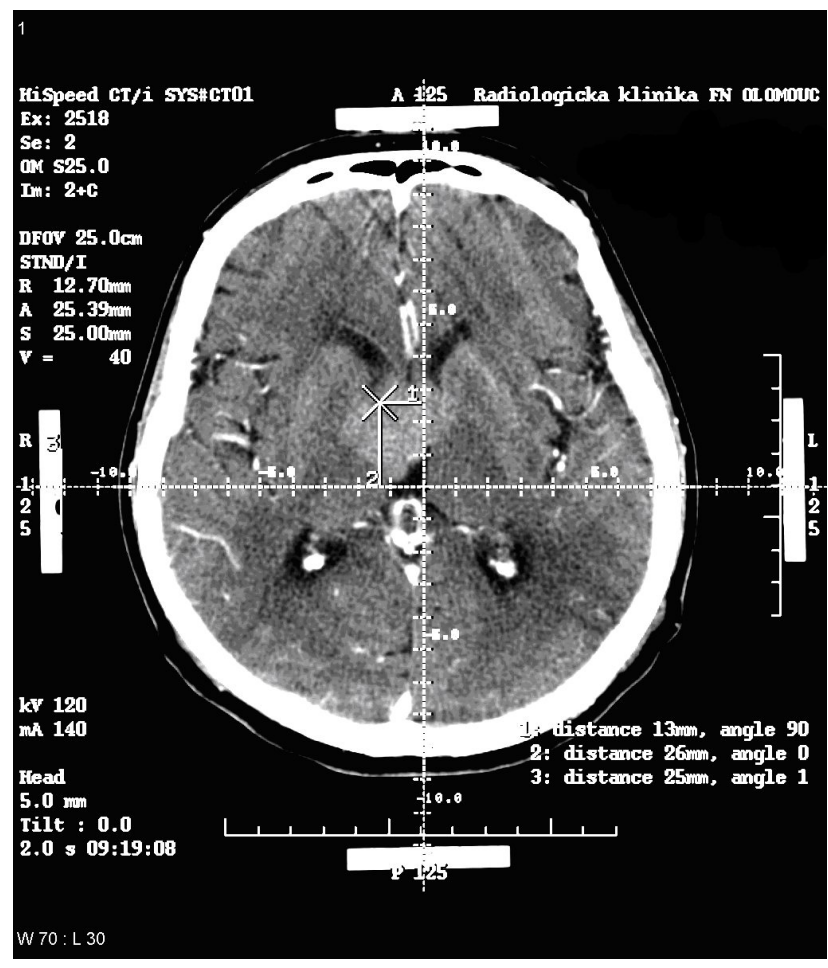

Fig. 1. CT-guided stereotactic biopsy.

120-240 mmol/day), $\mathrm{Cl}^{-}$(1215 mmol/day, normal 174$260 \mathrm{mmol} /$ day $)$ and $\mathrm{K}^{+}$(99 mmol/day, normal 35-80 $\mathrm{mmol} /$ day $)$ ions. Plasma renin values were low $(<0.1$, normal range 0.26-2.41 pmol/1) but aldosterone levels were within the normal range. Overall fluid balance was negative. The values of brain natriuretic peptide (NT-proBNP) were increased (631 ug/1, normal 1-93 ug/1), with no apparent previous history of cardiac disease. The patient was intensively hydrated in an effort to achieve balanced volumes and ions. Chemotherapy was initiated with highdose methotrexate according to DeAngelis protocol ${ }^{10}$.

After the first chemotherapy cycle, the CT scan confirmed regression of the tumour site to about one third of its original size. This was also accompanied by patient's improved cognition (Glasgow coma scale 14) as well as decreased polyuria and urine electrolyte excretion. Eventually, it was concluded that the diagnosis was cerebral salt wasting syndrome.

In patients with severe CNS disorders, disturbances of sodium metabolism are frequent. Distinction made between SIADH and CSW is of crucial importance for selecting adequate treatment strategy. The association between lymphomas involving deep structures of the brain and CSW that has not been described to date. The prevalence of the syndrome in hemato-oncological patients may be higher than commonly believed. Thus, it is necessary to investigate the causes of hyponatremia in patients with CNS lymphomas since early diagnosis and adjustment of the internal environment are a condition for subsequent intensive chemotherapy.

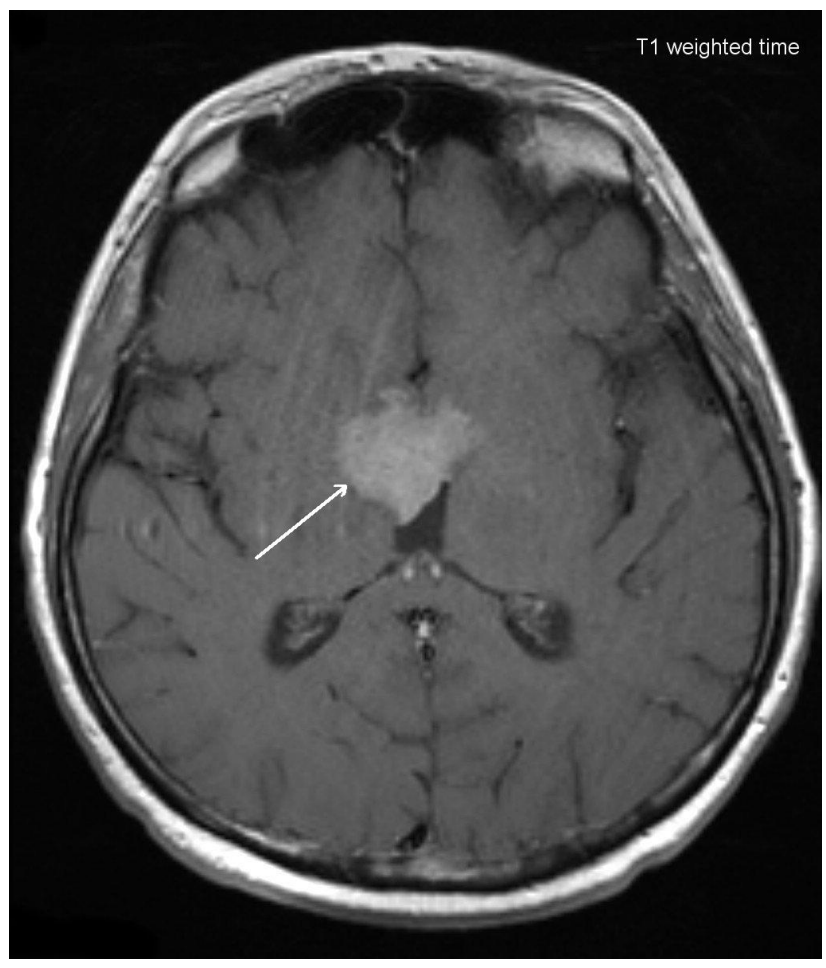

Fig. 2. Contrast magnetic resonance imaging, T1 weighted time.

\section{AKNOWLEDGEMENTS}

Supported by the grant of the Ministry of Education of the Czech Republic (MSM 6198959205).

\section{REFERENCES}

1. Peters JP et al. A salt-wasting syndrome associated with cerebral disease. Trans Assoc Am Physicians 1950;63:57-64.

2. Tisdall M, Crocker M, Watkiss J et al. Disturbances of sodium critically ill adult neurologic patients: a clinical review. J Neurosurg Anesthesiol 2006;18:57-63.

3. Fraser JF, Stieg PE. Hyponatremia in neurosurgical patient: epidemiology, pathophysiology, diagnosis and management. Neurosurgery 2006;59(2):222-9.

4. Safarova R, Horackova M, Vankova S et al. Central salt wasting diuresis syndrome as a cause of hyponatremia in patients at the internal medicine department. Vnitr Lek 2001;47:484-7.

5. Harrigan MR. Cerebral salt wasting syndrome. Crit Care Clin 2001;17:123-38.

6. Singh S, Bohn D, Carlotti A et al. Cerebral salt wasting: Truths, fallacies, theories, and challenges. Crit Care Med 2002;30:2575-79.

7. Palmer BF. Hyponatremia in patients with central nervous system disease: SIADH versus CSW. Trends Endocrinol Metab 2003;14:182-7.

8. Cerdà-Esteve M, Cuadrado-Godia E, Chillaron JJ. Cerebral salt wasting syndrome: review. European Journal of Internal Medicine 2008;19:249-254.

9. Carlotti AP, Bohn D, Rutka JT et al. A method to estimate urinary electrolyte excretion in patients at risk for developing cerebral salt wasting. J Neurosurg 2001;95:420-4.

10. Abrey LE, Yahalom J, DeAngelis LM. Treatment for primary CNS lymphoma: the next step. J Clin Oncol 2000;18:3144-50. 